\title{
Peroxidase obtida de farelo de soja aplicada a degradação de aflatoxinas B1 e M1 no leite
}

\author{
Francine Kerstner de Oliveira ${ }^{1}$, Ana Flávia Vendramin Comunello ${ }^{1}$, Lucielen Oliveira dos Santos ${ }^{2}$, Jaqueline Garda Buffon ${ }^{1}$
}

\begin{abstract}
Resumo
O leite é um alimento amplamente consumido na dieta humana por ser fonte de macro e micronutrientes. No entanto, este produto alimentício também se caracteriza pela frequente ocorrência de micotoxinas. Em vista disto, o uso de enzimas na degradação de micotoxinas vem sendo estudado com resultados promissores sendo observados quanto ao emprego da peroxidase. Neste contexto, o objetivo desse estudo foi empregar a enzima peroxidase extraída de farelo de soja visando a degradação de aflatoxinas $B_{1}$ e $M_{1}$ em leite sob condições de processamento. Para tanto, o extrato bruto de peroxidase obtido de farelo de soja (0,015 $U$ $\left.\mathrm{mL}^{-1}\right)$ foi adicionado ao leite que continha aflatoxinas $\mathrm{B}_{1}$ e $\mathrm{M}_{1}\left(5 \mathrm{ng} \mathrm{mL} \mathrm{L}^{-1}\right)$, mantendo sob refrigeração durante $24 \mathrm{~h}$, seguida da quantificação da concentração residual das micotoxinas. Como resultado foi observado que a peroxidase extraída de farelo de soja apresentou degradação média de $90,7 \%( \pm 0,3 \%)$ e $75,8 \%$ ( $\pm 7,1 \%)$ para aflatoxinas $\mathrm{B}_{1}$ e $\mathrm{M}_{1}$, respectivamente. O resultado obtido nesse estudo demonstra que a peroxidase extraída de farelo de soja pode ser uma alternativa de baixo custo para degradação de aflatoxinas $B_{1}$ e $M_{1}$ no leite, apresentando degradações superior a $75 \%$ mesmo sob condição de resfriamento, permitindo a sua aplicação durante transporte e processamento do leite.
\end{abstract}

Palavras-chave: enzima; micotoxina; mitigação.

\section{Introdução}

O leite é alimento fonte de macro e micronutrientes amplamente consumido em todo o mundo. As características nutricionais, bem como o $\mathrm{pH}$ neutro e a elevada atividade de água favorecem o desenvolvimento de contaminações microbianas no leite (SHARABI; OKUN; SHPIGELMAN, 2018), sendo o tratamento térmico o principal método utilizado para reduzir ou eliminar tais contaminações (CLAEYS et al., 2013). No entanto, os tratamentos térmicos não impedem que contaminantes termoresistentes estejam presentes no leite, tais como micotoxinas, que provém do consumo de ração contaminada pelo gado leiteiro (BRUERTON, 2001).

As micotoxinas são metabólitos tóxicos produzidos por fungos (BENNETT; KLICH, 2003). Dentre as micotoxinas, a aflatoxina $\mathrm{B}_{1}$ se destaca pela toxicidade (BIEHL; BUCK, 1987), sendo classificada pela International Agency for Research on Cancer (IARC) no Grupo 1 como carcinogênica a humanos (IARC, 2012). Essa micotoxina, assim como o seu principal metabólito, a aflatoxina $\mathrm{M}_{1}$ (BENNETT; $\mathrm{KLICH}, 2003)$, são amplamente encontrados em alimentos (GONÇALEZ et al., 2005), tendo destaque o leite, com níveis de ocorrência acima do regulamentado por órgãos fiscalizadores (GONÇALVES et al., 2018; SIBAJA et al., 2019).

Diferentes métodos têm sido avaliados a fim de reduzir os níveis de contaminação por micotoxinas em alimentos (BATA; LÁSZTITY, 1999). Entre os métodos, os biológicos têm se apresentado promissores para a degradação de micotoxinas, principalmente a aplicação de enzimas (FELTRIN, 2017), como a peroxidase (SIBAJA et al., 2018). Esta é uma enzima que está associada a reações de transferência de elétrons (NELSON; COX, 2014) e atua decompondo o peróxido de hidrogênio na presença de um doador de elétrons (VÁMOS-VIGYÁZÓ; HAARD, 1981). Essa enzima pode ser obtida de diferentes fontes, tais como coprodutos agroindustriais, se destacando o farelo de soja (OLIVEIRA; SANTOS; GARDA-BUFFON, 2021), por ser abundante devido a extração de óleo.

No leite, a potencial aplicação da peroxidase comercial para degradação de aflatoxinas B1 e M1 foi avaliada no estudo desenvolvido por Sibaja e colaboradores (2018). Os autores observaram a redução de $97 \%$ para aflatoxina B1 e $65 \%$ para aflatoxina $\mathrm{M} 1$, quando $0,015 \mathrm{U} \mathrm{mL}^{-1}$ de enzima foi usado e mantido a $30^{\circ} \mathrm{C}$ por $8 \mathrm{~h}$ (SIBAJA et al., 2018). Entretanto, a peroxidase comercial utilizada pelos autores é de elevado custo, devido a sua pureza. Ainda, a temperatura utilizada na etapa de degradação $\left(30^{\circ} \mathrm{C}\right)$ e o tempo $(8 \mathrm{~h})$ tornam sua aplicação em leite inviável, devido a perecibilidade desse produto. Neste contexto, o objetivo desse estudo foi empregar a enzima peroxidase extraída de farelo de soja visando a degradação de aflatoxinas B1 e M1 em leite sob condições de processamento.

\section{Material e Métodos}

O coproduto agroindustrial farelo de soja para extração da enzima foi obtido de empresa localizada na cidade de Rio Grande, RS, Brasil. Para padronizar a granulometria, a amostra foi moída e peneirada em peneiras tipo mesh tamanho 32 . O leite utilizado nos ensaios de degradação foi obtido do comércio local da cidade de Pelotas, RS, Brasil.

\footnotetext{
${ }^{1}$ Laboratório de Micotoxinas e Ciência de Alimentos, Escola de Química e Alimentos, Universidade Federal do Rio Grande (FURG). Avenida Itália km 8, Rio Grande, Rio Grande do Sul, Brasil. E-mail: frankerstner@gmail.com

${ }^{2}$ Laboratório de Biotecnologia, Escola de Química e Alimentos, Universidade Federal do Rio Grande (FURG). Avenida Itália km 8, Rio Grande, Rio Grande do Sul, Brasil.
} 
Os padrões das aflatoxinas $\mathrm{B}_{1}$ e $\mathrm{M}_{1}$ foram adquiridos da empresa Sigma Chemical Company (E.U.A.) e as soluções estoque foram preparadas pela dissolução dos padrões em benzeno:acetonitrila (98:2). A obtenção do extrato bruto de peroxidase do farelo de soja foi feito conforme descrito por Feltrin e colaboradores (2017).

A capacidade de degradação das aflatoxinas $B_{1}$ e $M_{1}$ no leite foi avaliada conforme condições descritas por Sibaja e colaboradores (2018) modificado. Para isso, as aflatoxinas $\mathrm{B}_{1}$ e $\mathrm{M}_{1}$ foram adicionadas ao reator perfazendo a concentração de $5 \mathrm{ng} \mathrm{mL} \mathrm{m}^{-1}$ e o solvente evaporado sob corrente de nitrogênio. Após, $5 \mathrm{~mL}$ da amostra de leite UHT (isentas de contaminação) foi adicionada, agitada em vórtex (30 s), banho ultrassônico (3 min) e mantida 30 min sob refrigeração. Posteriormente, foi adicionado $0,015 \mathrm{U} \mathrm{mL}^{-1}$ de peroxidase e $0,08 \%$ de $\mathrm{H}_{2} \mathrm{O}_{2}$, seguido da incubação sob refrigeração durante 24 h. Após o tempo de reação, as micotoxinas residuais no sistema foram extraídas e quantificadas. Experimento controle (sem a adição de enzima e na presença dos demais componentes de reação) foi realizado a fim de confirmar a ação da enzima na degradação das micotoxinas ou o efeito dos reagentes. Todos os ensaios de degradação foram realizados em triplicata.

A extração de ambas micotoxinas do leite foi feita segundo o método descrito por Gonçalves e colaboradores (2018). A quantificação das aflatoxinas $\mathrm{B}_{1}$ e $\mathrm{M}_{1}$ foi feita por cromatografia líquida com detector de fluorescência (LC-FL) e processadas no software LC Solution. A fase móvel foi constituída de água:metanol:acetonitrila $\left(60: 25: 15 \mathrm{v} \mathrm{v}^{-1}\right)$ com eluição isocrática. A determinação cromatográfica foi realizada empregando coluna

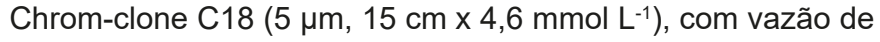
fase móvel de $1 \mathrm{~mL} \mathrm{~min}^{-1}$ e $40{ }^{\circ} \mathrm{C}$ de temperatura de forno. Os comprimentos de onda de excitação e emissão utilizados foram de 370 a $410 \mathrm{~nm}$, respectivamente, com volume de injeção de amostra de $20 \mu \mathrm{L}$ (SIBAJA et al., 2018). A identificação dos compostos foi feita com base no tempo de retenção e a concentração calculada a partir do pico obtido relacionado com a curva de calibração na matriz.

\section{Resultados e Discussão}

A Tabela 1 apresenta a curva analítica na matriz, bem como seus parâmetros analíticos, para quantificação das aflatoxinas $\mathrm{B}_{1}$ e $\mathrm{M}_{1}$ no leite.

Tabela 1: Parâmetros analíticos para quantificação de aflatoxina $B_{1}$ e $M_{1}$ em leite

\begin{tabular}{ccc}
\hline Parâmetros analíticos & Aflatoxina B1 & Aflatoxina M1 \\
\hline Curva analítica & $\mathrm{y}=4087,1 \mathrm{x}+4595,8$ & $\mathrm{y}=7041,7-8115,6$ \\
Faixa linear de trabalho $\left(\mathrm{ng} \mathrm{mL}^{-1}\right)$ & $3,0-15,0$ & $1,4-10,2$ \\
Coeficiente de determinação & 0,9838 & 0,9618 \\
Coeficiente de correlação & 0,9919 & 0,9807 \\
LOQ $\left(\mathrm{ng} \mathrm{mL}^{-1}\right)$ & 3,0 & 1,4 \\
LOD $\left(\mathrm{ng} \mathrm{mL}^{-1}\right)$ & 1,5 & 1,0 \\
\hline
\end{tabular}

LOQ: Limite de quantificação. LOD: Limite de deteç̧ão.
As recuperações médias $(n=3)$ determinadas a partir das curvas na matriz corresponderam ao intervalo de $85,4-95,7 \%$ para aflatoxina $B_{1}$ e $70,5-83,8 \%$ para aflatoxina $M_{1}$, portanto, em conformidade com o Regulamento (CE) n. ${ }^{\circ} 401$ da Comissão Europeia (EC, 2006).

As amostras de leite usadas para elaboração dos ensaios apresentaram contaminação natural por aflatoxina $\mathrm{M}_{1}(<\mathrm{LOQ})$ e ausência de aflatoxina $B_{1}$, estando de acordo com a Resolução n. ${ }^{\circ} 7$ da Anvisa (ANVISA, 2011).

Baseando-se nas concentrações de ambas as micotoxinas (Tabela 2), foi possível determinar o percentual de degradação das aflatoxinas $\mathrm{B}_{1}$ e $\mathrm{M}_{1}$ em leite. A peroxidase extraída de farelo de soja apresentou degradação média de $90,7 \%( \pm 0,3 \%)$ e $75,8 \%( \pm 7,1 \%)$ para aflatoxinas $B_{1}$ e $M_{1}$, respectivamente. Esse resultado é similar ao obtido por Sibaja e colaboradores (2018), que observaram a redução de $97 \%$ e $65 \%$ da concentração das aflatoxinas $B_{1}$ e $M_{1}$ no leite empregando a enzima peroxidase comercial $\left(0,015 \mathrm{U} \mathrm{mL}^{-1}\right)$ a $30{ }^{\circ} \mathrm{C}$, por $8 \mathrm{~h}$.

Tabela 2: Área do pico de aflatoxinas $\mathrm{B}_{1}$ e $\mathrm{M}_{1}$ em leite

\begin{tabular}{ccc}
\hline Ensaio & Micotoxina & Concentração $\left(\mathrm{ng} \mathrm{mL}^{-1}\right)$ \\
\hline Tratamento 1 & Aflatoxina B1 & 2,25 \\
& Aflatoxina M1 & 3,17 \\
\hline \multirow{2}{*}{ Tratamento 2 } & Aflatoxina B1 & 24,40 \\
& Aflatoxina M1 & 9,52 \\
\hline Tratamento 3 & Aflatoxina B1 & ND \\
& Aflatoxina M1 & 0,87 \\
\hline
\end{tabular}

Tratamento 1: Enzima + micotoxina. Tratamento 2: Micotoxina. Tratamento 3: Enzima. ND: Não detectado.

O resultado obtido nesse estudo mostra uma alternativa de baixo custo (peroxidase extraída de farelo de soja) para a degradação de aflatoxinas $B_{1}$ e $M_{1}$ no leite. Baseando-se nas condições de temperatura $\left(\approx 5^{\circ} \mathrm{C}\right)$ para atuação da enzima na degradação, sugere-se que a aplicação da peroxidase possa ser feita nas etapas de resfriamento (na fazenda produtora de leite), bem como nos tanques de transporte desse produto até a indústria. Dessa forma, a aplicação da peroxidase extraída de farelo de soja durante as etapas de processamento do leite pode ser uma alternativa para fornecer um alimento seguro ao consumidor.

\section{Conclusões}

A peroxidase extraída do coproduto agroindustrial farelo de soja apresentou capacidade de degradação de aflatoxinas $\mathrm{B}_{1}$ e $\mathrm{M}_{1}$ no leite, com percentuais de $90,7 \%$ e $75,8 \%$, respectivamente. Neste contexto, o emprego de peroxidase de baixo custo mostrou-se efetiva na degradação de aflatoxinas $B_{1}$ e $M_{1}$ em leite mantido sob refrigeração. 


\section{Referências}

ANVISA - AGÊNCIA NACIONAL DE VIGILÂNCIA SANITÁRIA. Resolução $n^{\circ} 7$, de 18 de fevereiro de 2011. Dispõe sobre limites máximos tolerados (LMT) para micotoxinas em alimentos. Disponível em: www.anvisa.gov.br. Acesso em: 26 abr. 2021.

BATA, A.; LÁSZTITY, R. Detoxification of mycotoxin-contaminated food and feed by microorganisms. Trends in Food Science \& Technology, v. 10, n. 6-7, p. 223-228, 1999.

BENNETT, J. W.; KLICH, M. Mycotoxins. Clinical Microbiology Reviews, v. 16, n. 3, p. 497-516, 2003.

BIEHL, M. L.; BUCK, W. Chemical contaminants: Their metabolism and their residues. Journal of Food Protection, v. 50 , n. 12, p. 1058-1073, 1987.

BRUERTON, K. Finding practical solutions to mycotoxins in commercial production: a nutritionist's perspective. In: Proceedings of the Alltech's 17th Annual Symposium, Queensland, Australia, p. 161-168, 2001.

CLAEYS, W. L.; CARDOEN, S.; DAUBE, G.; DE BLOCK, J.; DEWETTINCK, K.; DIERICK, K.; ZUTTER, L. D.; HUYGHEBAERT, A.; IMBERECHTS, H.; THIANGE, P.; VANDENPLAS, Y; HERMAN, L. Raw or heated cow milk consumption: Review of risks and benefits. Food Control, v. 31, n. 1, p. 251-262, 2013.

EC. European Commission. Commission Regulation N 401/2006 of 23 February 2006 laying down the methods of sampling and analysis for the official control of the levels of mycotoxins in foodstuffs. 2006.

FELTRIN, A. C. P.; FONTES, M. R. V.; GRACIA, H. D. K.; BADIALE-FURLONG, E.; GARDA-BUFFON, J. Peroxidase from soybean meal: obtention, purification and application in reduction of deoxynivalenol levels. Química Nova, v. 40, n. 8, p. 908-915, 2017.

GONÇALEZ, E.; FELICIO, J. D.; PINTO, M. M.; ROSSI, M. H.; NOGUEIRA, J. H. C.; MANGINELLI, S. Ocorrência de aflatoxina M1 em leite comercializado em alguns municípios do estado de São Paulo. Arquivos do Instituto Biológico, v. 72, n. 4, p. 435-438, 2005.
GONÇALVES, K. D. M.; SIBAJA, K. V. M.; FELTRIN, A. C. P.; REMEDI, R. D.; GARCIA, S. O.; GARDA-BUFFON, J. Occurrence of aflatoxins $\mathrm{B}_{1}$ and $\mathrm{M}_{1}$ in milk powder and UHT consumed in the city of Assomada (Cape Verde Islands) and southern Brazil. Food Control, v. 93, p. 260-264, 2018.

IARC (International Agency for Research on Cancer). Monographs on the evaluation of carcinogenic risks to humans: chemical agents and related occupations. IARC Scientific Publication, Lyon, France, v. 100, p. 224-248, 2012.

NELSON, D. L.; COX, M. M. Princípios de bioquímica de Lehninger. Porto Alegre: Artmed, 2014.

OLIVEIRA, F. K.; SANTOS, L. O.; GARDA-BUFFON, J. Mechanism of action, sources, and application of peroxidases. Food Research International, v. 143, p. 110266, 2021.

SHARABI, S.; OKUN, Z.; SHPIGELMAN, A. Changes in the shelf life stability of riboflavin, vitamin $C$ and antioxidant properties of milk after (ultra) high pressure homogenization: Direct and indirect effects. Innovative Food Science \& Emerging Technologies, v. 47, p. 161-169, 2018.

SIBAJA, K. V. M.; GARCIA, S. O.; FELTRIN, A. C. P.; REMEDI, R. D.; CERQUEIRA, M. B. R.; BADIALE-FURLONG, E.; GARDA-BUFFON, J. Aflatoxin biotransformation by comtmercial peroxidase and its application in contaminated food. Journal of Chemical Technology and Biotechnology, v. 94, n. 4, p. 11871194, 2018.

SIBAJA, K. V. M.; GONÇALVES, K. D. M.; GARCIA, S. O.; FELTRIN, A. C. P.; NOGUEIRA, W. V.; BADIALE-FURLONG, E.; GARDA-BUFFON, J. Aflatoxin $\mathrm{M}_{1}$ and $\mathrm{B}_{1}$ in Colombian milk powder and estimated risk exposure. Food Additives \& Contaminants. Part B, Surveillance, v. 12, n. 2, p. 97-104, 2019.

VÁMOS-VIGYÁZÓ, L.; HAARD, N. F. Polyphenol oxidases and peroxidases in fruits and vegetables. Critical Reviews in Food Science and Nutrition, v. 15, n. 1, p. 49-127, 1981. 